\title{
3 Research Square \\ The 12-year Experience - A Retrospective and Hospital-based Study of Gestational Trophoblastic Neoplasia in the Northwest of China
}

\section{Jiatao Hao}

The First Affiliated Hospital of Xi'an Jiaotong University

\section{Minghai Ma}

The First Affiliated Hospital of Xi'an Jiaotong University

Peipei Li

The First Affiliated Hospital of Xi'an Jiaotong University

\section{Luyuan Wang}

The First Affiliated Hospital of Xi'an Jiaotong University

\section{Hui Yu}

The First Affiliated Hospital of Xi'an Jiaotong University

\section{Yan Xue}

The First Affiliated Hospital of Xi'an Jiaotong University

\section{Ruifang An}

The First Affiliated Hospital of Xi'an Jiaotong University

Kexiu Zhu ( $\nabla$ KexiuZhu007@163.com )

Xi'an Jiaotong University Medical College First Affiliated Hospital

\section{Research Article}

Keywords: gestational trophoblastic neoplasia (GTN), invasive mole, choriocarcinoma, clinical characteristics, retrospective.

Posted Date: September 9th, 2021

DOl: https://doi.org/10.21203/rs.3.rs-780703/v1

License: (c) (i) This work is licensed under a Creative Commons Attribution 4.0 International License. Read Full License 


\section{Abstract}

Purpose: To analyze clinical features of gestational trophoblastic neoplasia (GTN) patients who received initial treatments at the First Affiliated Hospital of Xi'an Jiaotong University.

Methods: The patient record system was screened to extract clinical data including demographics, pregnancy history, diagnosis, staging, prognostic score, treatment. Student $t$ test, $x^{2}$ test, and Fischer's exact test were used.

Results: This study included 387 patients with GTN. Patients were divided into 2009-2014 and 2015-2020 groups, including 177 and 210 cases, respectively. Patients in 2015-2020 group had higher parity than those in 2009-2014 group (1.2 \pm 1.2 vs $0.9 \pm 1.2, P=0.030)$. Nullípara (73 [41.2\%] vs 65 [31.0\%]) and primípara (71 [40.1\%] vs 84 [40.0\%]) were more frequently seen in 2009-2014 group than those in 20152020 group, but multípara (61 [29.0\%] vs 33 [18.7\%]) was more common in 2015-2020 group ( $P=0.028)$. For patients in 2015-2020 group, more patients demonstrated 1 or more metastases (107 [51.0\%] vs 63 [35.6\%], $P=0.014$ ) than those in 2009-2014 group. Patients in 2015-2020 group had more distant invasions affecting more organs, including lungs (76 [36.2\%] vs 52 [29.4\%]) and lungs combining with other organs (31 [14.8\%] vs 11 [6.2\%]), than those in 2009-2014 group $(P=0.003)$.

Conclusions: Although patients diagnosed with GTN between 2015 to 2020 is associated with higher parity, more children, and more distant metastases, we cannot simply conclude that clinical features of GTN has changed over time.

\section{Introduction}

Gestational trophoblastic neoplasia (GTN), derived from the trophoblastic epithelium of the placenta, encompasses a heterogeneous range of malignant disorders, comprising invasive mole, choriocarcinoma, placental site trophoblastic tumor (PSTT) and epithelioid trophoblastic tumor (ETT) [1, 2]. These neoplastic conditions show varying histopathological and cytogenetic features as well as potential for local invasion and distant metastasis, and respond remarkably well to cytotoxic drugs [2]. Clinical features of GTN differ throughout the world, which could be attributable to differences in geographical patterns, disparity in definitions and diagnostic criteria, discrepancies between reference center-based and population-based data, or methodological difficulties in obtaining accurate data $[3,4]$. In North America and Europe, reference centers or national registries could improve availability of medical records by aggregating information of GTN patients, and standardization of diagnosis and treatment procedures $[1,4,5]$. Since 1973, all GTN patients in UK have been registered in the national disease centers for $\beta$-hCG surveillance, which can monitor malignant progression of the disease as well as therapeutic efficacy of chemotherapy [6, 7]. To elucidate better guidelines for treatment and follow-up, China has also set up hospital-based registries, such as Peking Union Medical College Hospital, for GTN patients in some regions [8-10]. However, hospital-based protocols for $\beta$-hCG monitoring and chemotherapy still exist variations, but with principles alike. Herein we conducted this retrospective study 
to analyze the clinical features of GTN patients treated at the First Affiliated Hospital of Xi'an Jiaotong University from 2009 to 2020.

\section{Materials And Methods}

\subsection{Patients}

We searched the computerized patient record system of the First Affiliated Hospital of Xi'an Jiaotong University for GTN patients between January 1st, 2009 and December 31st, 2020, using the keywords "invasive mole", "choriocarcinoma", "gestational trophoblastic neoplasia", and "gestational trophoblastic disease". 387 patients diagnosed with invasive mole and choriocarcinoma were finally collected in this study. All of them received initial chemotherapy in our hospital. All patients were analyzed by every 6 years (2009-2014 and 2015-2020). Medical records were screened to extract demographic characteristic, pregnancy history, symptom, diagnosis, metastatic condition, clinical stage, FIGO score, chemotherapy regimen, and auxiliary surgery. This study excluded patients with incomplete medical records, and with diagnosis of PSTT and ETT. The protocol of this study was approved by the Ethics Committee of the First Affiliated Hospital of Xi'an Jiaotong University. The informed consent was waived because this was a retrospective study.

\subsection{Initial assessment and diagnostic criteria of GTN}

When a diagnosis of GTN is suspected, an initial assessment including evaluation for risk factor and metastatic workup is undertaken. Along with the history-taking and physical examination, complete blood cell count, serum chemistries including renal and liver function panels, and quantitative serum $\beta$-hCG level were obtained. Because patients with GTN commonly manifest with vaginal bleeding or local invasiveness, transvaginal or transabdominal sonography was routinely used. Recommended radiologic methods include X-ray or computed tomography (CT) scan of the chest [11-12]. CT or magnetic resonance imaging (MRI) scan of brain, and abdomen and pelvis were performed if patients had neurological or abdominal symptoms. At our hospital, we followed the International Federation of Gynecology and Obstetrics (FIGO) criteria to diagnose GTN, which was defined in 2002 [13-15]. After molar evacuation, postmolar GTN is usually diagnosed with $\beta$-hCG surveillance in asymptomatic patients, although some patients present with irregular bleeding. Diagnosis of postmolar GTN is established if one of the following components was met: (1) $\beta$-hCG plateau for 4 consecutive values over 3 weeks; (2) 3 sequential rise of $\beta$-hCG value over 2 weeks; (3) persistent $\beta$-hCG value for 6 months; (4) histopathologic diagnosis of choriocarcinoma. With excluding residual pregnancy tissue or repregnancy, patients that may present with abnormal uterine bleeding were diagnosed with non-postmolar GTN if $\beta$ hCG value persistently elevated or sequentially rised over 4 weeks after abortion, term birth, or ectopic pregnancy. Notably, GTN is made based on $\beta$-hCG levels that fail to normalize, not necessarily focusing on histologic or pathological evidence.

\subsection{Treatments}


Indications for chemotherapy of GTN in our hospital include a plateaued or rising $\beta$-hCG value, or histological diagnosis of choriocarcinoma, which are similar to those recommended by FIGO guidelines and other disease centers. Gynecologic oncologists treated GTN based on the prognostic scoring system which predicts the potential for developing resistance to monochemotherapy and aids therapeutic choices. Before the first course of chemotherapy, patients were all scored. Combining prognostic scoring with anatomical staging, GTN patients were classified into low-risk group (stages I-III, score $<7$ ) and high-risk group (stage IV and stages II-III, score $\geq 7$ ) $[13,16]$. In our center, patients scoring 6 or less were treated with a monochemotherapy, including methotrexate and actinomycin $\mathrm{D}$, whereas some of those scoring 5 to 6 or diagnosed with choriocarcinoma might receive multi-agent chemotherapy. For patients with a score of 7 or more, several different multidrug regimens have been administered, including floxuridine + actinomycin D + vincristine (FAV), floxuridine + actinomycin D + methotrexate (FAM), floxuridine + etoposide + methotrexate (FEM), actinomycin D + etoposide + methotrexate (AEM), floxuridine + actinomycin D + etoposide + vincristine (FAEV), and etoposide + methotrexate + actinomycin $\mathrm{D}(\mathrm{EMA})$ alternating with cyclophosphamide + vincristine (CO). Salvage treatment for high-risk GTN patients included paclitaxel + etoposide alternating with paclitaxel + cisplatin (TE/TP). In addition, adjuvant surgery and radiation were an option for high-risk patients that present with distant metastases, for example, brain or liver. Consolidated chemotherapy is continued for at least 2 to 3 courses after first normalized $\beta$-hCG value.

\subsection{Follow-up and treatment outcome}

$\beta$-hCG level was monitored regularly at one-month intervals for 12 months to ensure remission. In this study, the final follow-up was performed in Jan 2021. Treatment outcome was categorized as complete remission and failure. Complete remission is defined as a normal serum $\beta$-hCG level for 3 consecutive weeks and then monthly for 1 year. Chemotherapy resistance manifest a plateaued $\beta$-hCG level of $\pm 10 \%$ over the course of 2 weeks or a re-elevation of at least one test of $\beta$-hCG level. Relapse is defined as a reelevation of $\beta$-hCG level after three normal weekly measurements, in the absence of a repregnancy [17, 18].

\subsection{Statistical analysis}

Statistical analysis was performed with SPSS 24.0 (IBM, Armonk, NY, USA). Baseline characteristics were described using mean (standard deviation), median value (range), and proportion (\%). Mean comparison was done using the Student $t$ test after variance comparison. A comparison of proportions was done using the $x^{2}$ test or Fischer's exact test as recommended. A $P$ value of $<0.05$ was considered significant.

\section{Results}

From January 1st, 2009 to December 31st, 2020, a total of 437 patients were diagnosed with GTN at the First Affiliated Hospital of Xi'an Jiaotong University. After excluding 25 patients with incomplete medical records, 7 patients with PSTT and ETT, and 18 patients received initial chemotherapy at other hospitals, 387 patients were diagnosed with invasive mole or choriocarcinoma in this study (Fig. 1). Mean age of 
387 patients at diagnosis was $32.4 \pm 9.1$ years, and 93 (24.0\%) were aged over 40 years. Of 177 patients included in 2009-2014 group, mean age was $31.8 \pm 9.1$ years, and $40(22.6 \%)$ were aged over 40 years. The 2015-2020 group had slightly older age with mean age being $32.9 \pm 9.0$ years and with $53(25.2 \%)$ of 210 patients aging over 40 years. Mean time of gravidity of 387 patients was $2.7 \pm 1.9$, of which 208 (53.7\%) had 1-2 gestations. Of 177 patients in 2009-2014 group, mean time of gravidity was $2.6 \pm 1.7$, and $103(58.2 \%)$ patients had $1-2$ gestations. Mean time of gravidity was $2.8 \pm 2.1$ for 210 patients in 2015-2020 group, and 1-2 gestations occurred in 105 (50.0\%) patients (Table 1).

Mean time of parity in 2015-2020 group was significantly higher than that in 2009-2014 group (1.2 \pm 1.2 vs $0.9 \pm 1.2, P=0.030)$. Nullípara (73 [41.2\%] of 177 vs 65 [31.0\%] of 210) and primípara (71 [40.1\%] of 177 vs 84 [40.0\%] of 210) were more frequently seen in 2009-2014 group than those in 2015-2020 group, and multípara (61 [29.0\%] of 210 vs 33 [18.7\%] of 177) was more commonly seen in 2015-2020 group, indicating that patients in 2015-2020 group have more children $(P=0.028)$. The most common type of antecedent pregnancy was postmolar disease (113 [63.8\%] of 177 vs 144 [68.5\%] of 210) followed by abortion (52 [29.4\%] of 177 vs 44 [21.0\%] of 210) and term or preterm gestation (12 [6.8\%] of 177 vs 22 [10.5\%] of 210) in both of 2009-2014 and 2015-2020 groups. A comparative analysis of intervals from index pregnancy in two groups was carried out. Interval less than 4 months was the most frequently seen in both groups (118 [66.7\%] of 177 vs 144 [68.6\%] of 210) (Table 1).

Hemorrhage (181 [46.8\%]) was the most common symptom for 387 patients in overall group, followed by enlarged uterus for gestational age (59 [15.2\%]), gastrointestinal symptoms (43 [11.1\%]), theca lutein cyst (29 [7.5\%]), and neurological symptoms (19 [4.9\%]). Near a third of patients in the overall group showed no symptoms or signs (113 [29.2\%]). Compared with 177 patients in 2009-2014 group, 210 patients in 2015-2020 group had slightly higher frequency of hemorrhage (79 [44.6\%] vs 102 [48.6\%], $P=0.502$ ), theca lutein cyst $(13$ [7.3\%] vs 16 [7.6\%], $P=1.000)$ and gastrointestinal symptoms $(19$ [10.7\%] vs 24 [11.4\%], $P=0.957$ ), and lower frequency of enlarged uterus for gestational age (31 [17.5\%] vs 28 [13.3\%], $P=0.318)$ and neurological symptoms (10 [5.6\%] vs $9[4.3 \%], P=0.702)$, with no significant statistics (P冈0.05) (Table 2).

Diagnoses for 387 patients were available, including 267 (69.0\%) cases of invasive mole and 120 (31.0\%) cases of choriocarcinoma. Compared with 2009-2014 group, 2015-2020 group had more invasive mole (122 [68.9\%] of 177 vs 145 [69.0\%] of 210) and less choriocarcinoma (55 [31.1\%] of 177 vs 65 [31.0\%] of 210), with no significant statistics ( $P=1.000)$ [Figure 2, Table 3]. Of 387 patients included, $261(67.4 \%)$ had tumors smaller than 30 millimeters, $45(11.6 \%)$ had tumors ranging from 30 to 50 millimeters, and 81 $(20.9 \%)$ had tumors bigger than 50 millimeters. The difference of tumor size between two groups was insignificant $(P=0.759)$. More patients in 2015-2020 group demonstrated more than one metastases (63 [35.6\%] of 177 vs 107 [51.0\%] of 210, $P=0.014$ ). Compared with patients in 2009-2014 group, more than half of patients in 2015-2020 group had distant organ invasions, including lungs (52 [29.4\%] of $177 \mathrm{vs}$ 76 [36.2\%] of 210) and lungs combining with other organs (11 [6.2\%] of 177 vs 31 [14.8\%] of 210), with significant statistics $(P=0.03)$. FIGO scores were 0 to 6 in $143(80.8 \%), 7$ to 12 in $28(15.8 \%)$, and 13 or 
more in 6 (3.4\%) patients in 2009-2014 group, compared with 166 (79.0\%), 35 (16.7\%), and 9 (4.3\%), respectively, of patients in 2015-2020 group, with no significant difference $(P=0.870)$ (Table 3).

Data for pretreatment $\beta$-hCG level in overall group included $84(21.7 \%)$ smaller than $10^{3}, 108(27.9 \%)$ ranging from $10^{3}$ to $10^{4}, 129(33.3 \%)$ ranging from $10^{4}$ to $10^{5}$, and $66(17.1 \%)$ greater than $10^{5}$. No difference was observed between two groups with respect to pretreatment $\beta$-hCG levels $(P=0.488)$. As for initial chemotherapy regimens applied to patients in two groups, single-agent and multi-agent regimen were given to $69(39.0 \%)$ vs $88(41.9 \%)$ and $108(61.0 \%)$ vs $122(58.1 \%)$ patients, respectively, with no significant between-group variance $(P=0.632)$. Mean chemotherapy cycles were similar between groups $(P=0.104)$, whereas 210 patients in 2015-2020 group used more initial chemotherapy cycles $(5.5 \pm 2.3)$ than those in $2009-2014$ group (5.1 \pm 2.6$) .89(23.0 \%)$ of 387 patients underwent adjuvant surgeries as part of their treatment. Patients in 2015-2020 group underwent less surgeries (41 [19.5\%] of 210) than those in 2009-2014 group (48 [27.1\%] of 177), with no significant difference ( $P=0.099)$ (Table 4).

\section{Discussion}

GTN is a malignant tumor that shows variable epidemiologic features and geographical patterns from published studies across the regions [3]. Data of choriocarcinoma from Asian countries were heterogeneous, ranging between 63 to 202 per 100,000 pregnancies in surveys from China, India, and Indonesia and below 23 per 100,000 pregnancies in studies from Japan and Singapore [3, 19-23]. In contrast with relatively high ratios of GTN found in these regions, a remarkably low ratio was found in Europe, North America, Australia, and some area of Latin America [3, 19, 20, 24, 25]. These regional differences of GTN data is unavoidable, which may, in part, be due to differences in calculating methods of disease ratio and histological classification. Although a considerable number of retrospective studies and surveys is published to describe the clinical features for GTN patients in China [9, 10], most reports are based on major hospitals that locate in the southeast, especially in some developed regions, and thus lack precise researches needed for a reliable assessment of GTN in under-developed regions.

GTN patients in the northwest of China were treated at different hospitals rather than in designated disease centers. The First Affiliated Hospital of Xi'an Jiaotong University is one of the largest disease centers in the northwest of China, which plays an important role for GTN patients. This study totally included 387 GTN patients from the First Affiliated Hospital of Xi'an Jiaotong University between 2009 and 2020. By analyzing differences of clinical features between 2009-2014 and 2015-2020 groups, we found that parity and metastatic conditions have changed over time. In this study, as much as 33 (18.7\%) of 177 patients before 2014 had at least two babies, 61 (29.0\%) of 210 patients had at least two babies after 2015, indicating an increased fertility desire among the patients in 2015-2020 group. This finding is consistent with a published Chinese study involving 291 patients, with a mean parity of $1.29 \pm 0.81$ and $0.90 \pm 1.00$ for choriocarcinoma patients in the 2005-2009 group and 2000-2004 group, respectively [26]. However, analyses were not conducted to know that children are born before or after they get GTN. Before patients getting GTN, the two-child policy in China that introduced in recent years may be a reason for increased parity among patients in 2015-2020 group [27]. After disease remission, scientific guidance 
regarding effects of chemotherapy regimen on pregnancy and timing of another pregnancy may increase opportunities for patients to successfully become pregnant. This study may not completely explain the difference in parity and delivery between two groups, but it is tempting to speculate that main reasons affecting the desire for pregnancy of patients in 2009-2014 group are not GTN itself.

Approximately $30 \%$ GTN cases have distant metastases at the time of initial assessment, most commonly to lungs ( $80 \%$ of patients), followed by vagina ( $30 \%)$, liver $(10 \%)$, and brain $(10 \%)$. Therefore, chest radiography is recommended for all patients with GTN at the time of diagnosis. Metastases in lungs can be detected on chest X-ray or CT scan and the latter one is much more sensitive to detect smaller lesions with greater accuracy [28]. However, a debate regarding whether CT scan is superior to chest X-ray in detecting lung metastases is ongoing and some previous studies found conflicting results $[11,12]$. For this reason, chest CT is not commonly used in patients with GTN. In this study, patients in 2015-2020 group have more distant invasions affecting organs, especially lungs, which may attribute to heavy use of CT scan that confuses pulmonary nodule with metastatic lesions. In lung cancer screenings with CT scan, the average nodule detection rate per round in randomized controlled trials and cohort studies ranged from $3-50 \%[29,30]$. Therefore, lung nodules might be misdiagnosed as metastatic lesions with evident invasive mole and choriocarcinoma [31, 32]. We, thus, cannot simply conclude that the time has changed features of GTN. However, this study could remain gynecologic oncologist and radiologist to distinguish metastatic lesions that originate from GTN between lung nodules.

GTN is a group of pregnancy-related malignancies with few data given their rarity that occurs in women of reproductive age. Some previous studies have confirmed that maternal age was a significant factor in the risk for molar pregnancies $[4,5,33]$. According to a Canadian study involving 428 women that were identified with gestational trophoblastic disease (GTD), the rates of hydatidiform mole were highest in both younger (less than 20 years old) and older age groups (30 or more years old) [33]. It was also noted that GTN was disproportionately higher in those at extremes of age including 10 to 19 years and 40 to 54 years, based on a recent study $[4,5]$. In our study, the mean age of GTN patients is 32.4 years, which is similar to the median age (32 years) at the start of treatment for patients in a UK study [34]. The proportion of patients aged over 40 years in 2015-2020 group was higher than that reported in GTN patients with low-risk scores ( $25.2 \%$ vs $10.3 \%$ [ [35], indicating a failure to apply contraception. Dantas et al. found that hormonal contraception, the most common contraceptive methods, does not increase the occurrence of postmolar GTN and the clinical progression of GTN or retard $\beta$-hCG normalization [36]. Therefore, when considering maternal age at the time of GTN diagnosis, the effects of age for clinical progression and another pregnancy should be concerned.

Hydatidiform mole was the most common type of antecedent pregnancy among GTN patients in this study, followed by abortion and term or preterm gestation. The composition of antecedent pregnancy of GTN patients has no significant differences between 2009-2014 and 2015-2020 groups. Although abortion has been found to be one of the most frequent antecedent pregnancy among patients with ultra high-risk GTN [9], this was not observed in our study because we did not divide patients into groups using a FIGO score. Invasive mole usually developed from hydatidiform mole, whereas choriocarcinoma could 
derive from other gestational types including miscarriage and term pregnancy, which may lead to differences in primary symptoms [1, 2]. Vaginal bleeding was the most typical and common symptoms, followed by enlarged uterus for gestational age, theca lutein cyst. Elevated $\beta$-hCG level and local invasions were main causes for vaginal bleeding and enlarged uterus for gestational age. Symptoms or signs vary with disease location. Patients with lung metastasis might have a combination of haemoptysis and shortness of breath, whereas those with brain metastasis might present with seizure or headache. Therefore, when considering clinical manifestations for reproductive women showing respiratory and nervous symptoms, we should consider the possibility of GTN.

The FIGO 2019 guideline recommended that GTN patients with FIGO score less than 7 would receive monochemotherapy [37]. In this study, 309 (79.8\%) of 387 patients scored less than 7, but only 157 (40.6\%) of 387 patients received single-agent chemotherapy, indicating that some cases with a FIGO score less than 7 were given a multi-drug chemotherapy. Although FIGO scoring system would be helpful for identifying low-risk patients who need single-agent chemotherapy, the following conditions warrant a switch to multi-drug chemotherapy, including poor response to initial therapy, significant elevation in $\beta$ hCG level, development of metastasis, or resistance to sequential single-agent chemotherapy regimens [37]. In this study, 230 (59.4\%) of 398 patients received multi-drug chemotherapy. In our center, FAM is the most used multi-drug regimen that given patients with a score ranging 5 to 6 , which was not displayed in this study. In our center, patients scoring 5 or over would be given multi-agent chemotherapy including FAV, FAM, FEM, AEM, FAEV, and EMA-CO. However, some regimens were not widely used and we will conduct a cohort study to compare these regimens. For patients with drug-resistant disease, surgical resection can aid cure and $\beta$-hCG level will drop rapidly if lesion has been completely removed.

Some limitations of our study should be mentioned. First, this is a retrospective study that has incomplete information and recall bias; for example, history of spontaneous abortions may have been ignored. Second, we cannot exclude an inherent selection bias linked to the retrospective nature of the analysis and multicenter origin of patients. Third, we did not include patients diagnosed before 2009 because the information of these patients was recorded using paper-based medical documents that is not comprehensive. Finally, there are also some confounding factors, such as socioeconomic policies.

Overall, the results of this study showed that demographics and clinical features of patients with GTN did not change over time, with the exception of parity and metastatic conditions which have increased in the period of 2015 to 2020. As such, parity condition and functions of imaging tests in diagnosing GTN patients should be re-analyzed in the future.

\section{Declarations}

\section{Funding}

This retrospective study was funded by the National Natural Science Foundation of China [No.81972428].

\section{Conflict of interest statement}


The authors declare that the research was conducted in the absence of any commercial or financial relationships that could be construed as a potential conflict of interest.

\section{Availability of data and material}

All of the patient data come from the hospital's case repository. To protect the privacy of patients, the raw data supporting the findings of this study are available from the corresponding author with the permission of the hospital.

\section{Code availability}

Not applicable.

\section{Authors' contributions}

Conceptualization, JH, RA, and KZ. Methodology, JH, MM, YX, and KZ. Formal analysis, JH and MM. Data curation, MM, PL, LW, and HY. Writing-original draft preparation, JH, MM, YX, RA, and KZ. Writing review and editing, JH, RA, and KZ. Supervision, $Y X, R A$, and $K Z$. All authors contributed to the preparation of this study and approved the final version.

\section{Ethics approval}

The studies involving human participants were reviewed and approved by the Medical Ethics Committee of the First Affiliated Hospital of Xi'an Jiaotong University, Xi'an, China. Written informed consent for participation was not required for this study. The informed consent for participation was waived because this was a retrospective study in accordance with the national legislation and the institutional requirements.

\section{Consent to participate}

Not applicable.

\section{Consent for publication}

Written informed consent for publication was obtained from all participants.

\section{Acknowledgments}

The authors also wish to acknowledge Jingyi Yang for her help of data collection. All authors have approved the final version of the article, including the authorship list.

\section{References}

1. Seckl MJ, Sebire NJ, Berkowitz RS (2010). Gestational trophoblastic disease. Lancet 376(9742):717729 
2. Shih leM (2007). Gestational trophoblastic neoplasia-pathogenesis and potential therapeutic targets. Lancet Oncol 8(7):642-650

3. Altieri A, Franceschi S, Ferlay J, Smith J, La Vecchia C (2003). Epidemiology and aetiology of gestational trophoblastic diseases. Lancet Oncol 4(11):670-678

4. Diver E, Richardson M, Liao Cl, Mann AK, Darcy KM, Tian C, Kapp DS, Chan JK (2021). Age and racial differences in the presentation of gestational trophoblastic neoplasia. Int J Gynecol Cancer 31(2):194-202

5. Tarney CM, Tian C, Craig ER, Crothers BA, Chan JK, Gist GD, Bateman NW, Conrads TP, Hamilton CA, Larry Maxwell G et al (2018). Relative Effects of Age, Race, and Stage on Mortality in Gestational Choriocarcinoma. Int J Gynecol Cancer 28(2):338-345

6. Sebire NJ, Foskett M, Short D, Savage P, Stewart W, Thomson M, Seckl MJ (2007). Shortened duration of human chorionic gonadotrophin surveillance following complete or partial hydatidiform mole: evidence for revised protocol of a UK regional trophoblastic disease unit. BJOG 114(6):760-762

7. Blagden SP, Foskett MA, Fisher RA, Short D, Fuller S, Newlands ES, Seckl MJ (2002). The effect of early pregnancy following chemotherapy on disease relapse and foetal outcome in women treated for gestational trophoblastic tumours. Br J Cancer 86(1):26-30

8. Jiang F, Yang K, Wan XR, Xiang Y, Feng FZ, Ren T, Yang JJ, Zhao J (2020). Reproductive outcomes after floxuridine-based regimens for gestational trophoblastic neoplasia: A retrospective cohort study in a national referral center in China. Gynecol Oncol 159(2):464-469

9. Kong Y, Yang J, Jiang F, Zhao J, Ren T, Li J, Wang X, Feng F, Wan X, Xiang Y (2017). Clinical characteristics and prognosis of ultra high-risk gestational trophoblastic neoplasia patients: $\mathrm{A}$ retrospective cohort study. Gynecol Oncol 146(1):81-86

10. Xiao C, Yang J, Zhao J, Ren T, Feng F, Wan X, Xiang Y (2015). Management and prognosis of patients with brain metastasis from gestational trophoblastic neoplasia: a 24-year experience in Peking union medical college hospital. BMC Cancer 15:318

11. Price JM, Lo C, Abdi S, Winter MC, Coleman RE, Tidy J, Hancock BW (2015). The Role of Computed Tomography Scanning of the Thorax in the Initial Assessment of Gestational Trophoblastic Neoplasia. Int J Gynecol Cancer 25(9):1731-1736

12. Darby S, Jolley I, Pennington S, Hancock BW (2009). Does chest CT matter in the staging of GTN? Gynecol Oncol 112(1):155-160

13. Lurain JR (2011). Gestational trophoblastic disease II: classification and management of gestational trophoblastic neoplasia. Am J Obstet Gynecol 204(1):11-18

14. Ngan HY, Bender H, Benedet JL, Jones H, Montruccoli GC, Pecorelli S; FIGO Committee on Gynecologic Oncology (2003). Gestational trophoblastic neoplasia, FIGO 2000 staging and classification. Int J Gynaecol Obstet 83 Suppl 1:175-177

15. Shaaban AM, Rezvani M, Haroun RR, Kennedy AM, Elsayes KM, Olpin JD, Salama ME, Foster BR, Menias CO (2017). Gestational Trophoblastic Disease: Clinical and Imaging Features. Radiographics 37(2):681-700 
16. Lurain JR, Casanova LA, Miller DS, Rademaker AW (1991). Prognostic factors in gestational trophoblastic tumors: a proposed new scoring system based on multivariate analysis. Am J Obstet Gynecol 164(2):611-616

17. Maestá I, Nitecki R, Desmarais CCF, Horowitz NS, Goldstein DP, Elias KM, Berkowitz RS (2020). Effectiveness and toxicity of second-line actinomycin $D$ in patients with methotrexate-resistant postmolar low-risk gestational trophoblastic neoplasia. Gynecol Oncol 157(2):372-378

18. Maestá I, Nitecki R, Horowitz NS, Goldstein DP, de Freitas Segalla Moreira M, Elias KM, Berkowitz RS (2018). Effectiveness and toxicity of first-line methotrexate chemotherapy in low-risk postmolar gestational trophoblastic neoplasia: The New England Trophoblastic Disease Center experience. Gynecol Oncol 148(1):161-167

19. Gestational trophoblastic diseases. Report of a WHO Scientific Group. World Health Organ Tech Rep Ser. 1983;692:7-81

20. Shanmugaratnam K, Muir CS, Tow SH, Cheng WC, Christine B, Pedersen E (1971). Rates per 100,000 births and incidence of choriocarcinoma and malignant mole in Singapore Chinese and Malays. Comparison with Connecticut, Norway and Sweden. Int J Cancer 8(1):165-175

21. Nakano R, Sasaki K, Yamoto M, Hata H (1980). Trophoblastic disease: analysis of 342 patients. Gynecol Obstet Invest 11(4):237-242

22. Baltazar JC (1976). Epidemiological features of choriocarcinoma. Bull World Health Organ 54(5):523-532

23. Srivannaboon S, Vatananusara C, Boonyanit S (1974). The incidence of trophoblastic disease in Siriraj Hospital. J Med Assoc Thai 57(11):537-542

24. Smith HO, Hilgers RD, Bedrick EJ, Qualls CR, Wiggins CL, Rayburn WF, Waxman AG, Stephens ND, Cole LW, Swanson M et al (2003). Ethnic differences at risk for gestational trophoblastic disease in New Mexico: A 25-year population-based study. Am J Obstet Gynecol 188(2):357-366

25. Ringertz N (1970). Hydatidiform mole, invasive mole and choriocarcinoma in Sweden 1958-1965. Acta Obstet Gynecol Scand 49(2):195-203

26. Sun R, Zhang Y, Zheng W, Tian Q, An R, Xue Y (2016). Clinical Characteristics of Gestational Trophoblastic Neoplasia: A 15-Year Hospital-Based Study. Int J Gynecol Cancer 26(1):216-221

27. Luo ZC, Liu X, Wang A, Li JQ, Zheng ZH, Guiyu S, Lou T, Pang J, Bai XL (2021). Obstetricians' perspectives on trial of labor after cesarean (TOLAC) under the two-child policy in China: a crosssectional study. BMC Pregnancy Childbirth 21(1):89

28. Frijstein MM, Lok C, van Trommel NE, Ten Kate-Booij MJ, Massuger $L$, van Werkhoven $E$, Short $D$, Aguiar X, Fisher RA, Kaur B et al (2020). Lung metastases in low-risk gestational trophoblastic neoplasia: a retrospective cohort study. BJOG 127(3):389-395

29. Bach PB, Mirkin JN, Oliver TK, Azzoli CG, Berry DA, Brawley OW, Byers T, Colditz GA, Gould MK, Jett JR, et al (2012). Benefits and harms of CT screening for lung cancer: a systematic review. JAMA 307(22):2418-2429 
30. Wender R, Fontham ET, Barrera E Jr, Colditz GA, Church TR, Ettinger DS, Etzioni R, Flowers CR, Gazelle GS, Kelsey DK et al (2013). American Cancer Society lung cancer screening guidelines. CA Cancer J Clin 63(2):107-117

31. Gasparri R, Sedda G, Brambilla D, Girelli L, Diotti C, Spaggiari L (2019). When a Differential Diagnosis Is Fundamental: Choriocarcinoma Mimicking Lung Carcinoma. J Clin Med 8(11):2018

32. Li X, Xu Y, Liu Y, Cheng X, Wang X, Lu W, Xie X (2019). The management of hydatidiform mole with lung nodule: a retrospective analysis in 53 patients. J Gynecol Oncol 30(2):e16

33. Altman AD, Bentley B, Murray S, Bentley JR (2008). Maternal age-related rates of gestational trophoblastic disease. Obstet Gynecol 112(2 Pt 1):244-250

34. Sita-Lumsden A, Short D, Lindsay I, Sebire NJ, Adjogatse D, Seckl MJ, Savage PM (2012). Treatment outcomes for 618 women with gestational trophoblastic tumours following a molar pregnancy at the Charing Cross Hospital, 2000-2009. Br J Cancer 107(11):1810-1814

35. Jareemit N, Horowitz NS, Goldstein DP, Berkowitz RS, Elias KM (2020). Outcomes for relapsed versus resistant low risk gestational trophoblastic neoplasia following single-agent chemotherapy. Gynecol Oncol 159(3):751-757

36. Dantas PRS, Maestá I, Filho JR, Junior JA, Elias KM, Howoritz N, Braga A, Berkowitz RS (2017). Does hormonal contraception during molar pregnancy follow-up influence the risk and clinical aggressiveness of gestational trophoblastic neoplasia after controlling for risk factors? Gynecol Oncol 147(2):364-370

37. Abu-Rustum NR, Yashar CM, Bean S, Bradley K, Campos SM, Chon HS, Chu C, Cohn D, Crispens MA, Damast S, et al (2019). Gestational Trophoblastic Neoplasia, Version 2.2019, NCCN Clinical Practice Guidelines in Oncology. J Natl Compr Canc Netw 17(11):1374-1391

\section{Tables}

Due to technical limitations, tables are only available as a download in the Supplemental Files section.

\section{Figures}




\begin{tabular}{|c|}
\hline $\begin{array}{c}\text { All medical records retrieved from } \\
\text { hospital database using prespecified } \\
\text { search keywords }(\mathrm{n}=1534)\end{array}$ \\
\hline
\end{tabular}

272 patients diagnosed with hydatidiform mole

\begin{tabular}{c}
\hline Patients diagnosed with GTD \\
$(\mathrm{n}=711)$ \\
\hline
\end{tabular}

\begin{tabular}{c}
\hline Patients diagnosed with GTD \\
$(\mathrm{n}=711)$ \\
\hline
\end{tabular}

768 duplicates

55 patients with incomplete medical records

Patients diagnosed with GTN

$(n=437)$

(1) 25 patients with incomplete medical records

(2) 7 patients diagnosed with PSTT or ETT

(3) 18 patients did not received initial treatments in our hospital

Patients diagnosed with invasive

mole or choriocarcinoma $(n=387)$

Figure 1

From January 1st, 2009 to December 31st, 2020, a total of 437 patients were diagnosed with GTN at the First Affiliated Hospital of Xi'an Jiaotong University. After excluding 25 patients with incomplete medical records, 7 patients with PSTT and ETT, and 18 patients received initial chemotherapy at other hospitals, 387 patients were diagnosed with invasive mole or choriocarcinoma in this study (Figure 1). 


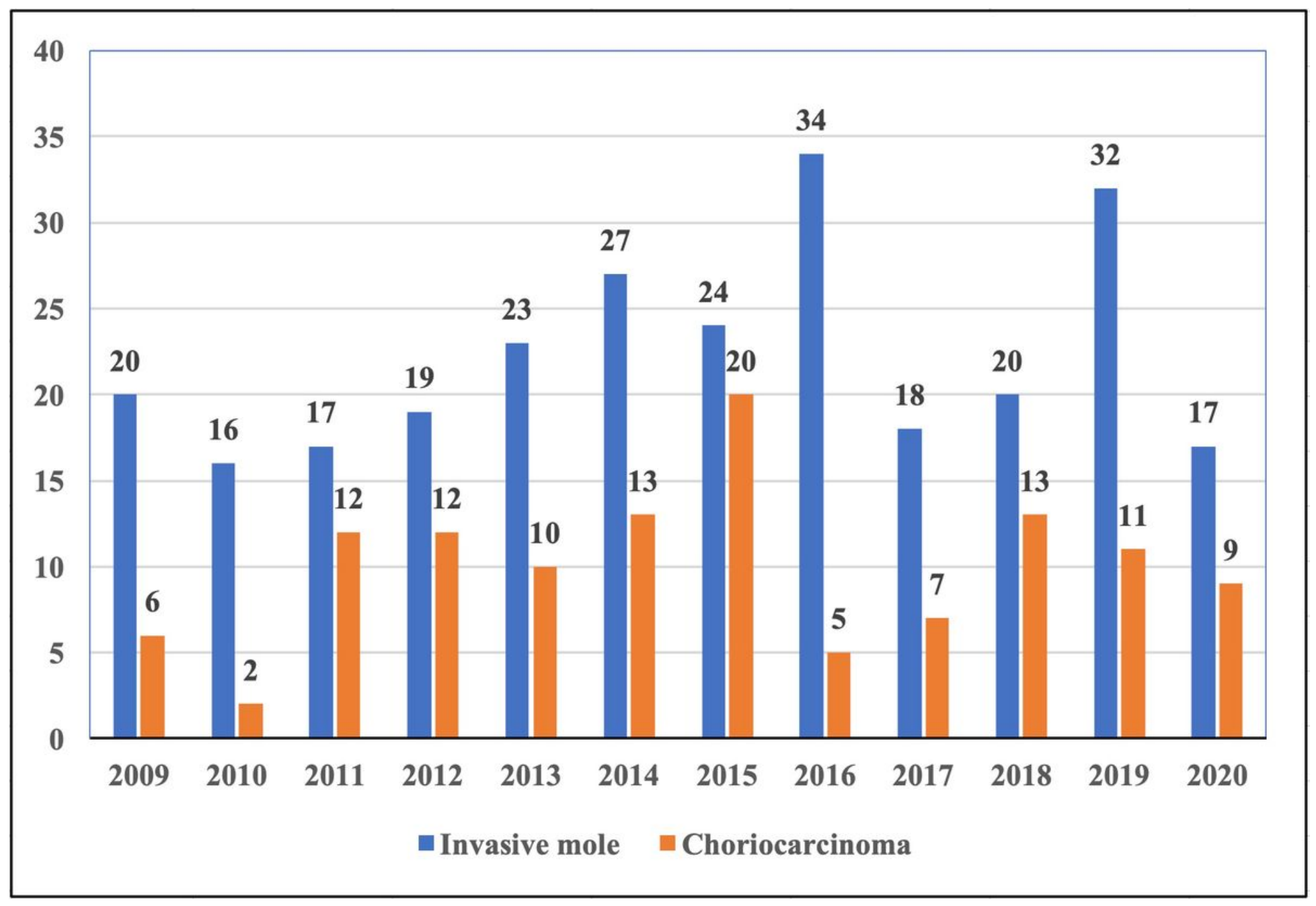

Figure 2

Diagnoses for 387 patients were available, including 267 (69.0\%) cases of invasive mole and 120 (31.0\%) cases of choriocarcinoma. Compared with 2009-2014 group, 2015-2020 group had more invasive mole (122 [68.9\%] of 177 vs 145 [69.0\%] of 210) and less choriocarcinoma (55 [31.1\%] of 177 vs 65 [31.0\%] of 210), with no significant statistics $(P=1.000)$ (Figure 2)

\section{Supplementary Files}

This is a list of supplementary files associated with this preprint. Click to download.

- Table1.xlsx

- Table2.xlsx

- Table3.xlsx

- Table4.xlsx 